\title{
EthoLog 2.2: A tool for the transcription and timing of behavior observation sessions
}

\author{
EDUARDO B. OTTONI \\ University of São Paulo, São Paulo, Brazil
}

\begin{abstract}
EthoLog is a tool that aids in the transcription and timing of behavior observation sessions - experimental or naturalistic, from video/audio tapes or registering real time. It was created with Visual Basic and runs on Windows $(3 . \mathrm{x} / 9 \mathrm{x})$. The user types the key codes for the predefined behavioral categories, and EthoLog registers their sequence and timing and saves the resulting data in ASCII output files. A sequential analysis matrix can be generated from the sequential data. The output files may be edited, converted to plain text files for printing, or exported to a spreadsheet program, such as MS Excel, for further analyses.
\end{abstract}

EthoLog is a tool that aids in the transcription and timing of behavior observation sessions-experimental or naturalistic, from video/audio tapes or registering real time. It was created with Visual Basic and runs on Windows $(3 . x / 9 x)$. The user types the key codes for the predefined behavioral categories, and EthoLog registers their sequence and timing and saves all the information for subsequent analyses. Many improvements have been made to the previous version (Ottoni, 1996): The whole interface has become more user friendly, there are more options for data output, and several new features have been added, such as an ethogram categories file editor, a sound alarm for fixed time intervals, a sequential analysis matrix generation tool, and an option for data input with a mouse.

\section{BASIC CONCEPTS AND GENERAL PROCEDURE}

\section{Defining the Ethogram}

Before transcribing the experimental or observational sessions, the user must create a file containing the ethogram - a list of behavioral categories and their key codes, the keys assigned to the behavioral categories. A key code is composed of only one character, which can be any letter (capitals included), symbol, or digit. The Categories Key Table files (ASCII files saved with the extension .ctg) are created or edited in the CTG files Editor window.

During transcription, the active categories' key codes are shown in the Behavioral Categories Listbox (Main window; Figure 1). EthoLog filters the keyboard input according to the key codes list in the loaded .ctg file, so

Correspondence concerning this article should be addressed to E. B. Ottoni, Department of Experimental Psychology, Institute of Psychology, University of São Paulo, Av. Prof. Mello Moraes 1721, Bloco A, Cidade Universitaria, S. Paulo, SP, Brazil, CEP 05508-900 (e-mail: ebottoni@usp.br). only the listed keys are active. Nonlisted characters typed are registered in the Keyboard Input field, but the program otherwise ignores them - unless a Label (see below) is being entered.

\section{State Events and Instant Events}

EthoLog deals with state events (SEs) and instant events (IEs). A key code preceded by a spacebar press is registered as an SE change; otherwise, the category occurrence is considered an IE. SEs have a duration. The starting time is registered when the spacebar is pressed, and the Current State Event field displays a “...?" until a behavioral category key code is entered (pressing the spacebar again, instead of a key code, cancels the previous press, and the last-entered SE goes on). There is always an SE going on; an SE ends when the user closes it or when another SE starts. In Version 2.2, however, a builtin NoState "category" was added: It constitutes the initial SE, and the user can return to it (when an SE ends without another user-defined SE starting) by pressing the spacebar (SE change) and the Escape key. IEs do not have durations, but times of occurrence. An IE can occur "inside" an SE in progress. Since SEs and IEs are only distinguished by the previous spacebar press (SEs), any behavioral category in the Categories Key Code Table can be used as an SE or an IE (or both).

\section{The Transcription Session}

After loading the Categories Key Code file to be used, the user must enter the name (prefix) to be given to the output files; before starting the session, some options can be set: State Repetition (allowed or not; determines whether an SE can be immediately followed by another SE of the same behavioral category), NoState (allowed or not; determines whether an SE can be be closed without another SE starting), Case Insensitive keyboard (on or off) Mouse Input (allowed or not; data input using the mouse instead of the keyboard), Session Duration (preset timer or undefined duration), and Timer Bell (a sound 


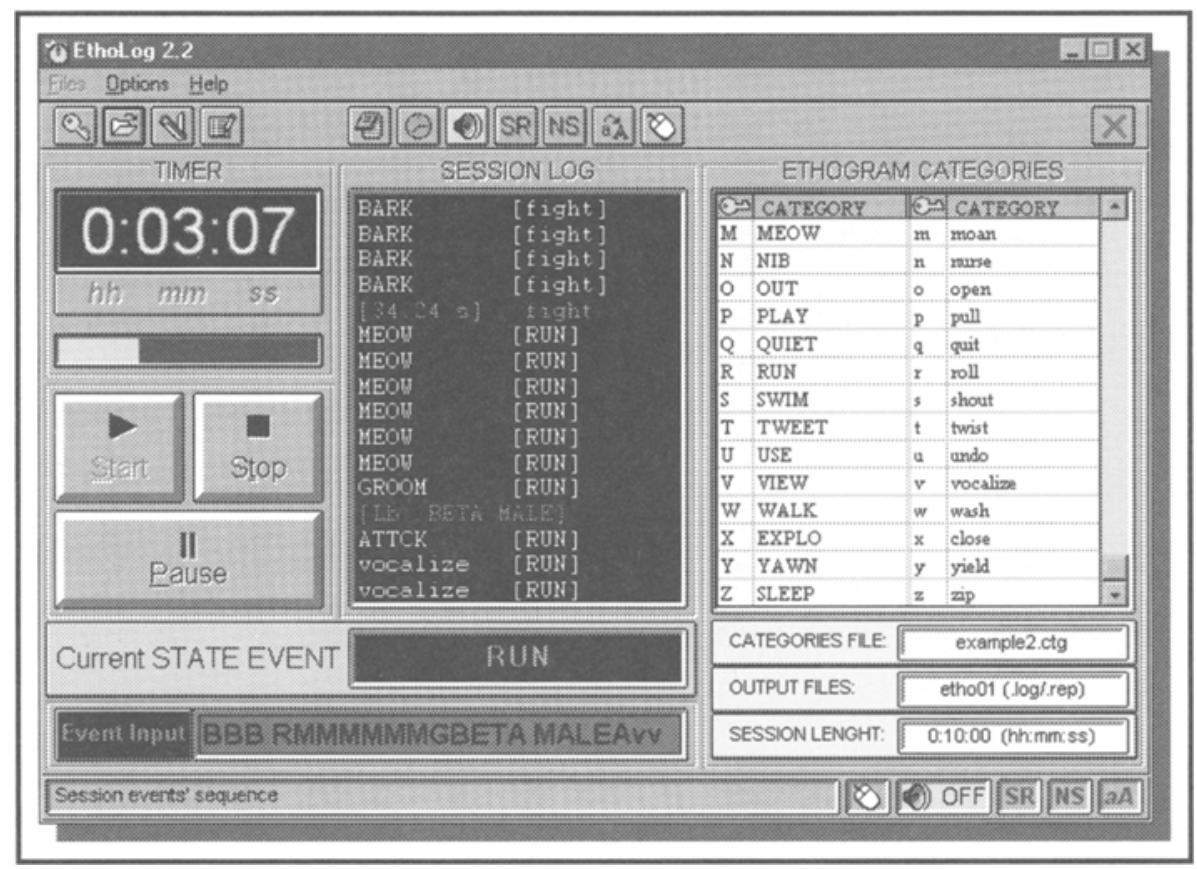

Figure 1. EthoLog 2.2 main window.

alarm) interval. Session Description Notes can be appended to the output files (to register information on the subject, procedures, experimental conditions, etc.).

The session can then be initiated by clicking the Start button. If the session duration was preset, the Elapsed Time Display in the Main window will be activated. The transcription can be interrupted and resumed by clicking the Pause/Resume button. Labels (notes or modifiers, up to 255 characters) can be appended to the last-entered event (Label inputs are initiated and closed by pressing the Enter key).

A session is closed when the user clicks the Stop button or when the Preset Timer reaches the predefined session duration. EthoLog generates and saves two output files containing the resulting sequential and timing data, which can afterward be displayed and edited in the Toolpack window (see below).

\section{THE OUTPUT FILES}

The output files (with the extensions .rep and .log) contain the complete data of the transcription session. They show the sequence of events and their durations (SEs) or times of occurrence (IEs), total SEs' durations and SE of occurrence of IEs, and the Labels for any SE or IE. The .rep file summarizes the occurrence of the behavioral categories (as SEs or IEs), and the .log file contains the complete events' sequence. These files can be viewed or edited (particular events or whole categories) in the Toolpack window. The Toolpack also allows the generation of Sequential Analysis (.seq; see below) files and Merged Log files (.lg2), which contain the same data as the original .log files, but with the IEs' and SEs' sequences intertwined in a single Events Sequence. All these files (.log, .rep, .lg2, and .seq) can be saved as plain text files for printing or exporting to a word processor, as well as exported to a spreadsheet program, such as MS Excel.

\section{Toolpack Window}

The Toolpack window (Figure 2) initially displays (after loading the selected output files) some general information about the transcription session and two tabbed panels with summaries of the .rep (Session Report panel) and .log files (Session Log panel). After a sequential analysis matrix is generated (see below), it is displayed in a third panel.

\section{Editing the Output Files}

Output files (.rep/.log) can be edited in two ways: editing individual IEs or SEs (and their Labels) or editing all occurrences of a given behavioral category. Session description notes can also be edited. The Alteration Notes box (Report panel) displays a summary of changes made to the original output files. These notes are saved in a field of the edited .rep and .log files.

SEs and IEs (and their associated Labels) can be individually edited (or deleted) by double-clicking them in the Session Log panel. The changes are immediately reflected in the totals displayed in the Session Report panel.

Deleting IEs just removes them from the list; deleting SEs replaces them by a NoState event. If the State Repetition Allowed box is checked, contiguous SEs of the same category as the edited SE are left as they are; if the 


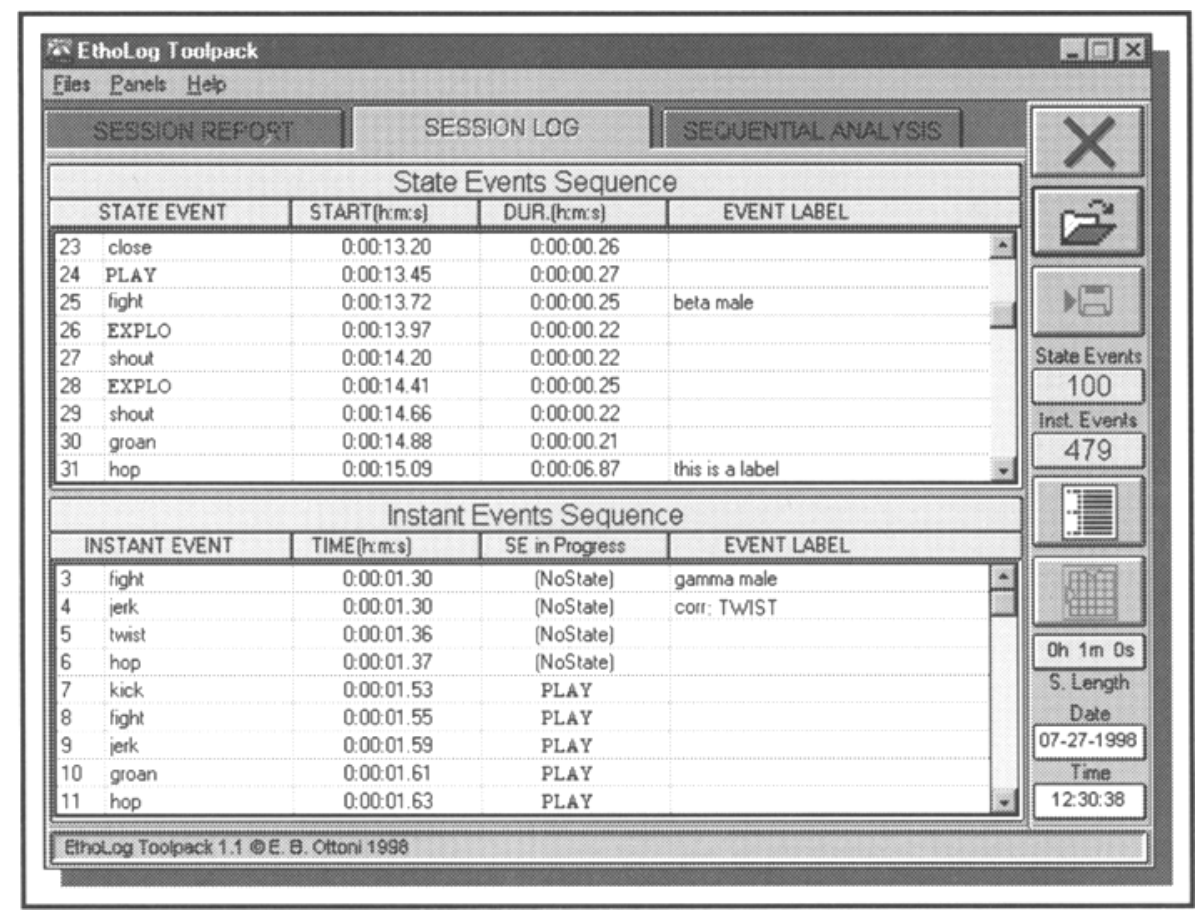

Figure 2. EthoLog Toolpack window.

box is unchecked, these SEs are joined (durations and Labels' contents added up). The only exception are contiguous NoState intervals, which are joined anyway.

Double-clicking a category name in the Report panel allows the user to edit it - to merge all occurrences of this behavioral category into another (for instance, the user may decide to pool all occurrences of the hypothetical categories walk, run, and locomotion into locomotion). The target category must exist in the original ethogram listed in the .ctg file. This option also allows the deletion of all occurrences of a behavioral category.

\section{Sequential Analysis}

Clicking the Sequential Analysis button generates a file (with the .seq extension) containing the sequential analysis matrix, which shows the occurrences of each IE category in the $\log$ file as preceding (antecedent to) or following (subsequent to) another IE category and the total occurrence of each behavioral category as an antecedent or a subsequent IE.

\section{Exporting the Output Files}

The output files generated by EthoLog can be easily exported to a spreadsheet program for further analyses or can be converted to plain text files for printing or exporting to a word processor. To open them with the MS Excel, the user must select, in the Import Assistant, the option(s) "file delimited by commas," with double quotes (") as text qualifiers.

\section{FINAL CONSIDERATIONS}

\section{Utilization Tips}

EthoLog includes an extensive Help file with detailed explanations on its usage. The program, in the present version, is more suited to observation of a focal subject. Labels applied to SEs or IEs can be used to specify other individuals interacting with the focal subject and/or the direction of the interaction (in case of "social" categories). Dyadic interactions can be directly transcribed with the use of Labels to indicate the agent or with the use of behavioral categories specific for each individual; assigning the same letter in capital and lowercase as the key code for the same category by each of the subjects allows alternating the focus on the subjects by means of the Caps Lock key (this works only with IEs). The behaviors of three or more interacting individuals can be transcribed for all the subjects, one at a time, and the individual output files can be brought together afterward and sorted in an integrated spreadsheet file for further analyses.

\section{Timers}

The program does not use the standard Visual Basic (VB) timer, since it is not accurate at the level of hundredths of a second and is affected by other events occurring in the Windows environment. Instead, EthoLog uses the TIMERINFO routine in TOOLHELP.DLL, which avoids these problems. Thus, even if there are delays in the updating of the Screen Timer (the "stopwatch" 
display), owing to other events in the Windows environment, the real timing is unaffected, and the Screen Timer still keeps the exact time, since the only part played by the regular VB Timer Control is reading the (correct) time from TIMERINFO once every tenth of a second.

\section{Availability}

There is, to our knowledge, one other behavioral data collection program available in the market--Noldus' The Observer, which is generally more powerful than EthoLog (allowing, for instance, data collection through hand-held devices), but also quite expensive. EthoLog Version 2.2 probably fits the needs of most behavioral observation transcription procedures and is distributed as freeware. The most recent release can be downloaded from its homepage on the Internet (http://www.geocities. com/ebottoni/ethohome.html or http://members.xoom. $\mathrm{com} /$ EthoLog/ethohome.html). Any comments or suggestions on future improvements are welcome.

\section{REFERENCE}

OtToni, E. B. (1996). EthoLog 1.0: Ethological transcription tool for Windows. Behavior Research Methods, Instruments, \& Computers, 28, 472-473.

(Manuscript received August 24, 1999; revision accepted for publication March 27, 2000.) 